\title{
The Single-Item Measure of Stress Symptoms after Myocardial Infarction and Its Association with Fatigue
}

\author{
Ulla Fredriksson-Larsson 1,2,3, Eva Brink ${ }^{1,2,3}$, Gunne Grankvist ${ }^{4}$, Ingibjörg H. Jonsdottir ${ }^{5,6}$, \\ Pia Alsen 1 \\ ${ }^{1}$ Department of Nursing, Health and Culture, University West, Trollhättan, Sweden \\ ${ }^{2}$ Institute of Health and Care Sciences, University of Gothenburg, Gothenburg, Sweden \\ ${ }^{3}$ Centre for Person-Centred Care, University of Gothenburg \\ ${ }^{4}$ Department of Social and Behavioural Studies, University West, Trollhättan, Sweden \\ ${ }^{5}$ Institute of Stress Medicine, Sahlgrenska Academy, University of Gothenburg, Gothenburg, Sweden \\ ${ }^{6}$ Institute of Neuroscience and Physiology, Sahlgrenska Academy, University of Gothenburg, Gothenburg, Sweden \\ Email: ulla.fredriksson-larsson@hv.se
}

Received 20 March 2015; accepted 10 April 2015; published 16 April 2015

Copyright (C) 2015 by authors and Scientific Research Publishing Inc.

This work is licensed under the Creative Commons Attribution International License (CC BY). http://creativecommons.org/licenses/by/4.0/

(c) (i) Open Access

\section{Abstract}

Surviving a myocardial infarction (MI) can be a stressful event entailing challenges in daily life during the recovery period. Experiencing fatigue symptoms post-MI has been described as bothersome and occurs in nearly half of patients four months and two years after MI. The aetiology of fatigue disorder is unclear, but research has shown that fatigue plays an important role in the relationship between stress and perceived poor health. Previous findings indicate that having access to an easily administered stress measurement is worthwhile both in the clinic and in research. The single-item measure of stress symptoms has not been validated in persons treated for MI. The aim was to validate the single-item measure of stress symptoms and to explore its association with fatigue in a sample of persons treated for MI. Methods: 142 respondents completed the questionnaires of the Multidimensional Fatigue Inventory-20, the single-item measure of stress symptoms and the Perceived Stress Scale-10 (PPS-10) two months post-MI. Correlation analysis and t-tests were used to validate the single-item stress measure and its association with post-MI fatigue. Results: The convergent validity of the single-item measure of stress symptoms was confirmed. In analyses of relations between stress and fatigue, it was found that the single-item stress measure was strongly associated with both the global fatigue score and all four fatigue dimension scores (general, physical and mental fatigue as well as reduced activity). Conclusion: The single-item measure of stress symptoms was found to be a valid measure of post-MI stress. Also, the measure was useful in assessing associations between stress and fatigue and could therefore indicate that 
post-MI fatigue experiences should be further explored in full using multidimensional fatigue assessment.

\title{
Keywords
}

\author{
Myocardial Infarction, Fatigue, Stress, Symptom Research
}

\section{Introduction}

Surviving a myocardial infarction (MI) can be a stressful experience that entails challenges in daily life during the recovery period [1]. In research on the relations between chronic stress and adverse outcomes post-MI, moderate to high stress was associated with almost double increased risk of dying compared to low levels of stress [2]. Also, persons who experienced moderate to high stress during the months preceding their MI showed increased probabilities of having angina one year after discharge [2]. There is a lack of research on the influence of stress on post-MI fatigue, but associations between perceived stress and fatigue have been identified in the general population [3]. Because post-MI stress seems to be insufficiently explored, it would be worthwhile to validate an easily administered stress symptom assessment measure and its association with fatigue in a sample of persons treated for MI.

\section{Background}

Stress is frequently implicated in medically unexplained fatigue [4], but there is still limited evidence for the role of stress following MI [5] and its association with post-MI fatigue has not yet been fully explored. During the recovery process after coronary heart disease, a large proportion persons have to deal with fatigue [6]. "Fatigue is an unpleasant symptom that incorporates bodily feelings ranging from tiredness to exhaustion, creating an unrelenting overall condition that interferes with persons' ability to function to their normal capacity” (p. 527) [7]. Fatigue has been described as a bothersome and common symptom post-MI [8] and has been reported in nearly half of the group four months and two years post-MI [9] [10]. Fatigue has generally been conceptualized as a warning sign indicating a harmful accumulation of stress [11]. One study also showed that fatigue played an important mediating role in the relationship between stress and perceived poor health [12]. High perceived stress, mediated by impaired sleep quality, contributed to fatigue in healthy adults [13]. There are many potential stressors that may be related to acute treatment and long-term recovery post-MI. Initially, persons are often admitted to a coronary care unit with symptoms of, for example, chest pain, sweating, nausea, shortness of breath, fatigue, dizziness or collapse [14]. Some persons described these life-threatening feelings as a "sense of dread" [15]. During recovery, some persons had to deal with emotional symptoms related to re-experiencing the MI, such as nightmares and psychological distress when they are reminded of the event [16]. They could experience anxiety related to insecurity about their future health and longevity [17] and emotional distress such as helplessness [18]. Also, acute stress symptoms and pain as well as hostility could predict vulnerability to stress disorder post-MI [19]. Other factors found to be stressful during recovery from MI involved daily struggles, i.e., how well persons managed to return to full-time work, experiences of extreme tiredness, and the fact that they had to stop smoking [20]. The subjective experience of the seriousness of MI rather than the objective seriousness predicted self-reported stress post-MI [21].

A single-item measure of stress symptoms has previously been used in research on associations between stress and injury among industry employees [22], and as a screening tool for assessing the degree of perceived stress in relation to physical activity and aerobic fitness [23]. Moreover, in a study on physical activity and mental health, the item was used to define a group with high perceived stress levels [24].

\section{Aim}

Given previous findings showing that perceived stress is an important health issue in patients with MI, it is of great interest to identify an easily administered stress measure that can be used for both clinical and research purposes. In the present study, the single-item measure of stress symptoms was validated in a sample of persons 
treated for MI. We also assessed whether this single measure is related to symptoms of fatigue in a sample of persons treated for MI.

\section{Method}

\subsection{Procedures}

The sample included 264 consecutive persons, recruited by three research nurses during the first week of treatment for MI from March 2011 to March 2012 in one coronary care unit in a Swedish rural hospital. The diagnostic criteria for MI were based on electrocardiographic, symptom and enzymatic criteria. Seventy-two persons were excluded due to cognitive disorientation, age over 75 years, communicative disabilities, and other severe diseases (e.g., cancer); 24 persons declined participation at baseline and three were missed and not asked. Two months after discharge, a number of questionnaires were completed by 142 respondents out of the 165 persons included at baseline (11 persons did not responded despite a reminder, 2 had died, 4 were excluded due to serious disease and 6 declined to participate) (Notable, one respondent was missing in measurement of fatigue). Thus the response rate was $86 \%$ at follow-up two months post-MI. The study was approved by the Regional Ethical Review Board in Gothenburg (720-10). The respondents were informed about the study both verbally and in writing. Verbal and written informed consent were obtained from those who agreed to participate in the study.

\subsection{Questionnaires}

The single-item measure of stress symptoms was used to assess symptoms of stress. The question was: "Stress means a situation when a person feels tense, restless, nervous, or anxious, or is unable to sleep at night because his or her mind is troubled all the time. Do you feel that kind of stress these days?” The response was made on a 5-point Likert scale varying from 1 "not at all” to 5 "very much" [25]. To construct a dichotomous scale, the response categories 1 - 3 were grouped to represent a low stress level and the response categories 4 - 5 indicated a high stress level [24]. The Perceived Stress Scale (PSS-10) is a 10-item questionnaire designed to measure "the degree to which situations in one's life are appraised as stressful” [26] (p. 385). The responses were made on a scale from "never" (0) to "very often" (4) during the past month. The measured items are general in nature [26] and are based on the stress-related components of unpredictable, uncontrolled and overwhelming life events [27]. A summarized (global) high score indicates a high stress level. A validation study showed good reliability and construct validity for the Swedish version [28]. In the present study, Cronbach's alpha was 0.88.The Multidimensional Fatigue Inventory (MFI-20) is a 20-item questionnaire originally developed to assess fatigue in five dimensions [29]. The response scale runs from agreement "yes, that is true" to disagreement "no, that is not true" on a scale from 1 - 5, where higher scores indicate more fatigue within a possible range of 20 - 100. In the present study, the MFI-20 was applied in accordance with results from a new validation study in a sample of persons treated for MI four months earlier. This meant measurement of an underlying global dimension (a summarized transformed global score) and multidimensional assessments of four of the five original dimensions: general fatigue, physical fatigue, reduced activity, and mental fatigue [30]. In the present study, Cronbach's alpha was between 0.88 and 0.92 .

\subsection{Data Analysis}

SPSS statistical software version 21 was used. Descriptive statistics included means, standard deviations (SD), medians, and analytic statistics comprised correlation analyses. Both parametric (Pearson) and nonparametric (Spearman) correlations were calculated, however, no major discrepancies were found. The results from Pearson's correlation coefficients are reported throughout, including the confidence interval for the single-item measure of stress symptoms. The convergent validity of the single-item measure of stress symptoms was examined by calculating the correlations with the PSS-10 global score as well as with all 10 separate items. Correlational analyses were also used to assess relations between the single-item measure of stress symptoms and the global and the multidimensional fatigue scores. Finally, an independent $t$-test was conducted with the singleitem measure of stress symptoms as the dichotomous variable and the global fatigue score as the continuous variable. 


\section{Results}

As showed in Table 1, there were fewer women (23\%) than men and the mean age of the persons was 63 years \pm 8.3 (range $35-75$ ). The majority of persons (81\%) were cohabiting, and $44 \%$ returned to work after MI. Symptom characteristics in the study population are presented in Table 2.

The convergent validity of the single-item measure of stress symptoms was confirmed by significant correlations, $0.315-0.665$ ( $\mathrm{p}<0.001)$, with both the global PSS-10 score and all separate items of the PSS-10; see Table 3. The correlation analyses showed that the single-item measure of stress symptoms was associated, $r=$ 0.512 (CI: 0.37 - 0.66) ( $<$ 0.001), with the global fatigue score and all four fatigue dimension scores (general fatigue, physical fatigue, reduced activity, and mental fatigue), $r=0.414-0.600$; see Table 4 . Using the square correlation coefficient, one obtains a value that can be interpreted in percent. That is, $26 \%$ of the variance in the global fatigue score could be explained by stress. The correlation results have also been presented in a box plot diagram, showing a monotonic relationship ( $\mathrm{y}$ increases as $\mathrm{x}$ increases, but the curve is not completely linear); see Figure 1. The response alternative "no stress at all" showed a wide range of variation in fatigue, meaning

\begin{tabular}{cc} 
Table 1. Demographics and clinical data $(\mathrm{N}=142)$. \\
\hline Age, mean (SD) and range & $35-75$ \\
Women, n (\%) & $63(8.3)$ \\
Intervention, n (\%) & $32(23)$ \\
Coronary Artery Bypass Graft, n (\%) & $5(3.5)$ \\
Beta-blockers n (\%) & $116(82)$ \\
Smoking, n (\%) & $12(8.4)$ \\
Cohabit, n (\%) & $115(81)$ \\
Occupational employed, n (\%) & $62(44)$ \\
\hline
\end{tabular}

Table 2. Descriptive data of fatigue and stress symptoms.

\begin{tabular}{|c|c|c|}
\hline Symptom & Mean (SD) & Range \\
\hline Stress (single item) & $2.10(1.04)$ & $1-5$ \\
\hline Fatigue global $^{1}$ & $59.15( \pm 11.72)$ & $20-89$ \\
\hline General fatigue $^{1}$ & $10.9(4.36)$ & $4-16$ \\
\hline Physical fatigue $^{1}$ & $10.6(4.44)$ & $4-16$ \\
\hline Reduced activity $^{1}$ & $10.7(4.36)$ & $4-16$ \\
\hline Mental fatigue $^{1}$ & 8.7 (4.03) & $4-16$ \\
\hline PSS total score & 11.39 (6.69) & $0-30$ \\
\hline PSS 1 & $1.06(0.84)$ & $0-4$ \\
\hline PSS 2 & $1.04(1.06)$ & $0-4$ \\
\hline PSS 3 & $1.24(0.93)$ & $0-4$ \\
\hline PSS 4 & 1.05 (1.08) & $0-4$ \\
\hline PSS 5 & $1.27(0.85)$ & $0-4$ \\
\hline PSS 6 & 1.35 (1.07) & $0-4$ \\
\hline PSS 7 & $1.39(1.01)$ & $0-4$ \\
\hline PSS 8 & $1.04(0.91)$ & $0-4$ \\
\hline PSS 9 & $1.18(0.97)$ & $0-4$ \\
\hline PSS 10 & $1.18(0.97)$ & $0-4$ \\
\hline
\end{tabular}

PSS: Perceived Stress Scale, $1 \mathrm{~N}=141$. 
Table 3. Pearson correlations between the single item of stress symptoms and the Perceived Stress Scale (PSS-10).

\begin{tabular}{ccc}
\hline PSS item & Correlation $^{\mathbf{1}}$ & $\boldsymbol{p}$-value \\
\hline PSS 1 & 0.444 & $<0.001$ \\
PSS 2 & 0.485 & $<0.001$ \\
PSS 3 & 0.665 & $<0.001$ \\
PSS 4 & 0.402 & $<0.001$ \\
PSS 5 & 0.377 & $<0.001$ \\
PSS 6 & 0.379 & $<0.001$ \\
PSS 7 & 0.434 & $<0.001$ \\
PSS 8 & 0.430 & $<0.001$ \\
PSS 9 & 0.345 & $<0.001$ \\
PSS 10 & 0.315 & $<0.001$ \\
PSS Global scale & 0.569 & $<0.001$ \\
\hline
\end{tabular}

${ }^{1}$ Pearson's correlation.

Table 4. Correlations between the single-item of measure of stress symptoms and fatigue $(\mathrm{N}=141)$.

\begin{tabular}{ccc}
\hline Fatigue & correlation $^{\mathbf{1}}$ & $\boldsymbol{p}$-value \\
\hline General fatigue & 0.584 & $<0.001$ \\
Physical fatigue & 0.423 & $<0.001$ \\
Reduced activity & 0.414 & $<0.001$ \\
Mental fatigue & 0.600 & $<0.001$ \\
Global fatigue & 0.512 & $<0.001$ \\
\hline
\end{tabular}

${ }^{1}$ Pearson's correlation.

that quite a few persons who scored high on the global fatigue score also reported low stress levels. Moreover, the response alternatives "to some degree" and "quite a lot of stress" showed small variation, but a high level of fatigue, meaning that persons who chose these response alternatives probably experienced high global fatigue. These findings were supported when the single-item measure of stress symptoms was treated as a dichotomous scale with wider spread in low stress levels; see Figure 2. The independent $t$-test analysis revealed a significant difference in fatigue levels between the group with high stress and the group with low stress, Table 5.

\section{Discussion}

The main results of the present study showed that a single-item measure of stress symptoms was associated with the well-validated PSS questionnaire, indicating that this single-item measure could be used to assess stress in persons treated for MI. There are many reasons for assessing and recognizing stress symptoms after treatment for MI. Stress is obviously a risk factor for coronary heart disease [5], and it contributes to pathophysiological changes and alterations in cardiac regulation that affect health outcomes [31]. Increased fear of dying and experiences of helplessness predicted stress status better than objective measures of MI severity did [18]. In interviews with women post-MI, it was found that they struggled to maintain control, and thus stress was generated when they experienced loss of control over daily life activities [20]. Also, persons who experienced moderate to high stress during the months preceding their MI showed increased probabilities of having angina one year after discharge and increased odds of having diminished quality of health [2]. Because MI can be a frightening and 


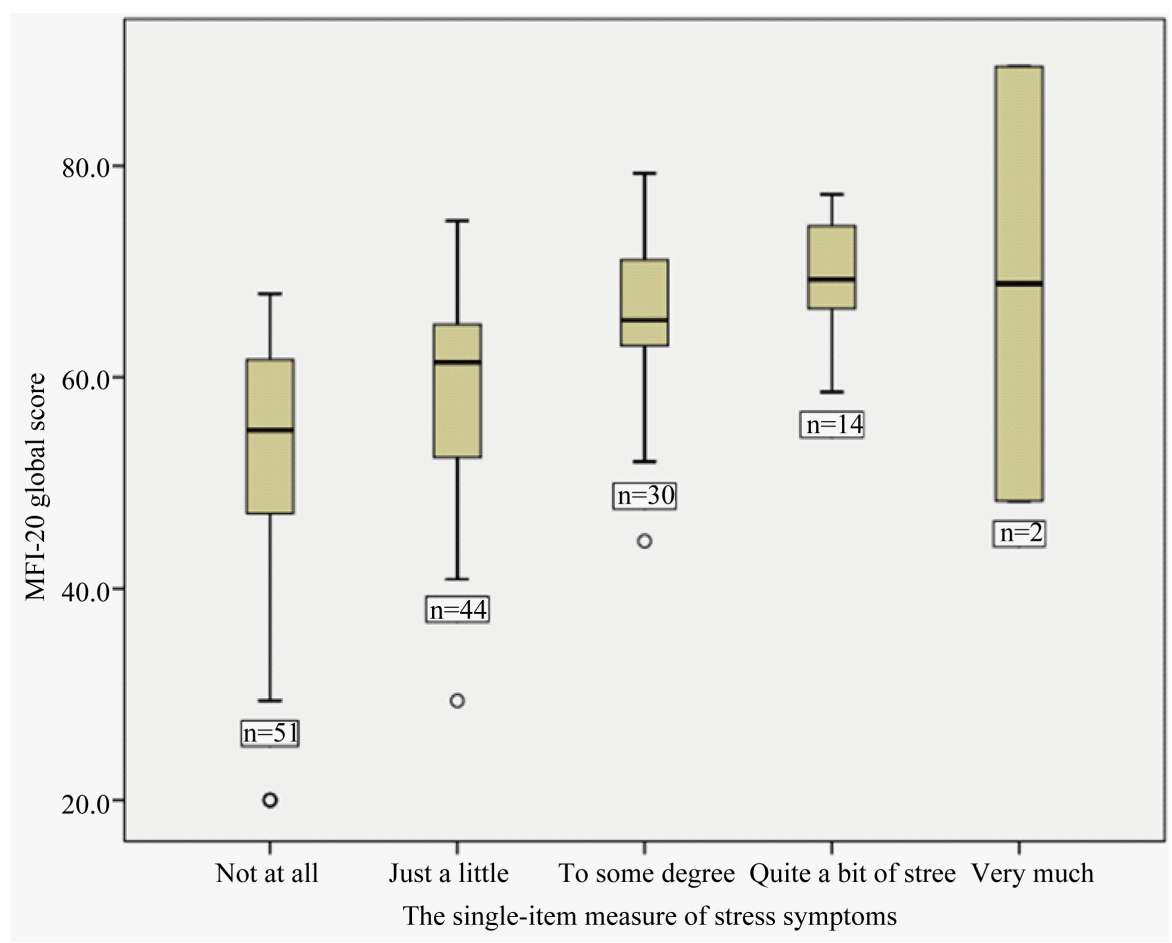

Figure 1. Correlation analyses between MFI-20 global score and the single-item measure of stress symptoms.

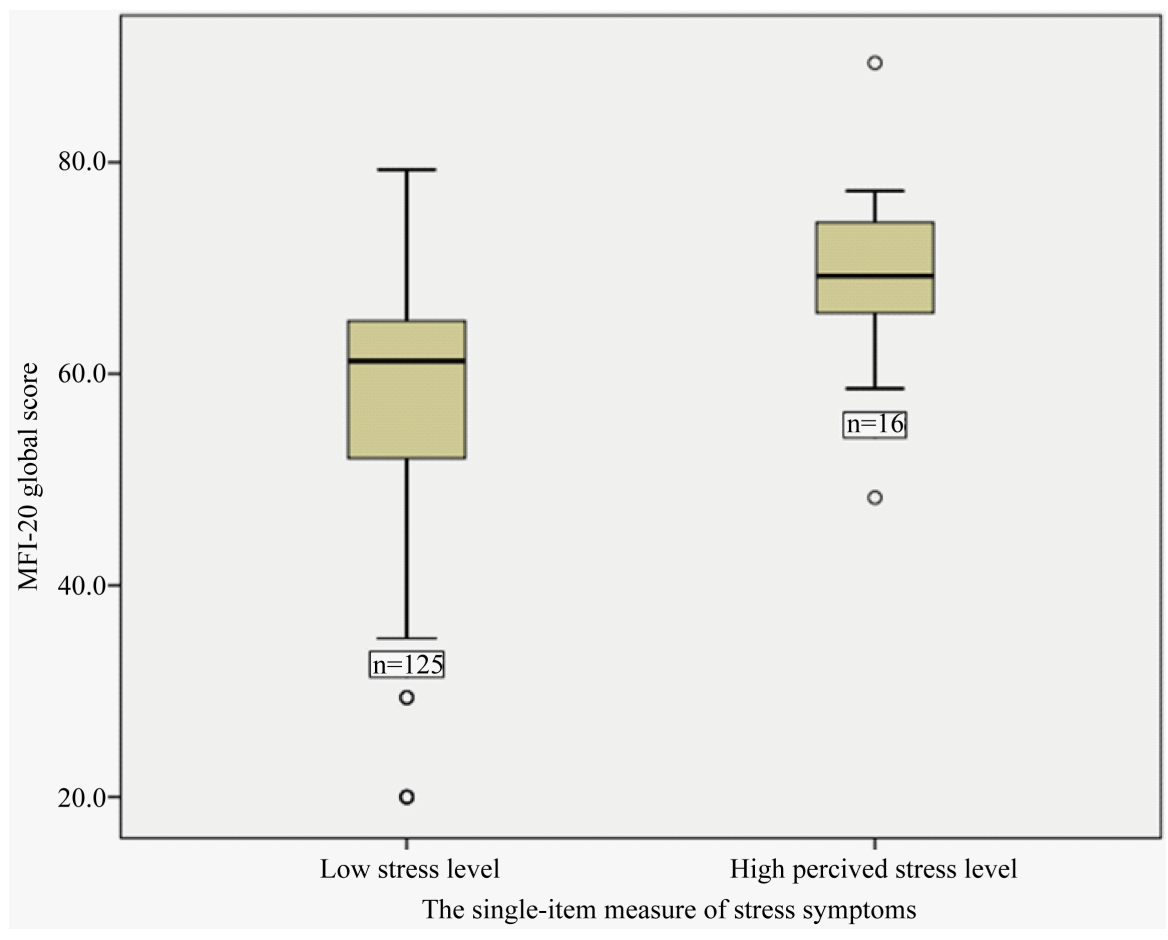

Figure 2. Correlation analyses between MFI-20 global score and the single-item measure of stress symptoms (dichotomous scale).

distressing experience [32], it would seem to be of vital importance to identify persons at risk for experiencing stress during recovery from MI. The present results showed that the single-item measure of stress symptoms was 
Table 5. Independent t-test between fatigue and stress.

\begin{tabular}{cccc}
\hline & $\mathrm{N}=141$ & Mean fatigue (SD) & t-test \\
\hline Stress yes & 16 & $69.34(11.40)$ & \\
Stress no & 125 & $57.85(9.13)$ & \\
Total & 141 & $59.15(11.72)$ & $-3.873 /$ CI $(-14.3-5.63)$ \\
t-test & & & 0.00 \\
$p$-value & & & 0.09 \\
Mean differences, 95\% & & & \\
\hline
\end{tabular}

associated with all of the separate PSS-10 items and with the PSS-10 global score, indicating that the single-item measure showed convergent validity. Using this single-item measure of stress symptoms may be advantageous given its simplicity, low cost, and ease of interpretation. The question was interpreted as being appropriately formulated, which should increase the probability that respondents will understand it correctly and respond as requested, in this case with regard to stress symptoms [33].

Moreover, the results showed that experience of stress measured by the single-item measure of stress symptoms correlated with the global score of the MFI-20. This means that higher stress levels were associated with higher fatigue levels. This is in line with a study of persons in a non-clinical sample, which showed that high stress levels were related to fatigue severity [13]. In the present sample, the single-item measure of stress symptoms was strongly correlated with all four fatigue dimensions of the MFI-20 (general, mental, physical fatigue and reduced activity) two months post-MI. The direction of the relationship between stress and fatigue is not always clear. In the present study, stress has been hypothesized to cause fatigue, but the opposite direction of causality is also possible, i.e., fatigue has been conceptualized as a warning sign indicating harmful accumulation of stress [11]. The mechanisms underlying the association between stress symptoms and fatigue are as yet unknown [34]. The present study indicates that a single-item stress measurement administered two months postMI could be used to identify persons at risk for negative stress experiences. Studies have shown that stress levels can change over time post-MI, and therefore, it would seem to be worthwhile to measure stress longitudinally during the recovery period [14]. Early and periodic identification of persons who are at risk for experiencing high stress levels could provide opportunities to personalize support by including stress management in the rehabilitation programme. This could also help in preventing the onset of fatigue or an increase in existing fatigue.

In our analyses of the validity of the single-item measure of stress symptoms, we treated the item as both a continuous and a dichotomous variable and found a significant relationship with fatigue in both cases. However, when using the item dichotomously, we identified a risk for neglecting the fact that some persons responding "no stress at all” could nonetheless be experiencing significant fatigue. In addition, 50\% of those who responded "stress to some degree" scored above the total sample median for the global fatigue score. However, this problem should be further investigated by, for example, analysing agreement between the measurements using a statistical method for analysis of paired ordinal data [35]. Also, persons recovering from MI may experience several symptoms, and the present study shows that exploring stress and fatigue simultaneously is justified. According to [36] Miaskowski et al. (2004), symptom management research should evaluate more than one symptom at a time as well as the relationships between symptoms. Therefore, future research could focus on the prevalence of post-MI stress and its relationship with other concurrent symptoms in addition to fatigue.

The present study was cross-sectional and this could be seen as a limitation because stress level can change over time post-MI. Therefore, further validation of the single-item measure of stress symptoms is warranted. Also, the relationship between stress and fatigue has not been explored regarding cause and effect, as such an analysis requires a more complex research design. However, one strength of the study is that the sampling procedure was consecutive, that is, all patients with MI admitted to a rural coronary care unit during one year were invited to participate. Consequently, we consider that the present result contributes knowledge about measurement of post-MI stress and its association with fatigue among persons ( $<75$ years) treated for MI in a rural area.

\section{Conclusion}

The single-item measure of stress symptoms was found to provide valid measures of post-MI stress. Moreover, 
the item was useful in measuring associations between stress and fatigue and can therefore indicate fatigue experiences. Further research should focus on stress and its relationship with other concurrent symptoms post-MI, in addition to fatigue.

\section{Conflicts of Interest}

No conflict of interest has been declared by the authors.

\section{Acknowledgements}

This work was supported by the Centre for Person-Centred Care at University of Gothenburg (GPCC), Sweden. The GPCC is funded by the Swedish Government's grant for Strategic Research Areas, Care Sciences (Application to Swedish Research Council no. 333-02) and co-funded by the University of Gothenburg, Sweden.

\section{References}

[1] Dullaghan, L., Lusk, L., McGeough, M., Donnelly, P., Herity, N. and Fitzsimons, D. (2014) “I Am Still a Bit Unsure How Much of a Heart Attack It Really Was!” Patients Presenting with Non ST Elevation Myocardial Infarction Lack Understanding about Their Illness and Have Less Motivation for Secondary Prevention. European Journal of Cardiovascular Nursing, 13, 270-276. http://dx.doi.org/10.1177/1474515113491649

[2] Arnold, S.V., Smolderen, K.G., Buchanan, D.M., Li, Y. and Spertus, J.A. (2012) Perceived Stress in Myocardial Infarction: Long-Term Mortality and Health Status Outcomes. Journal of the American College of Cardiology, 60, 17561763. http://dx.doi.org/10.1016/j.jacc.2012.06.044

[3] Kocalevent, R.D., Hinz, A., Brähler, E. and Klapp, B.F. (2011) Determinants of Fatigue and Stress. BMC Research Notes, 4, 238. http://dx.doi.org/10.1186/1756-0500-4-238

[4] van Houdenhove, B. and Egle, U.T. (2004) Fibromyalgia: A Stress Disorder? Psychotherapy and Psychosomatics, 73, 267-275. http://dx.doi.org/10.1159/000078843

[5] Steptoe, A. and Kivimäki, M. (2013) Stress and Cardiovascular Disease: An Update on Current Knowledge. Annual Review of Public Health, 34, 337-354. http://dx.doi.org/10.1146/annurev-publhealth-031912-114452

[6] Eckhardt, A.L., DeVon, H.A., Piano, M.R., Ryan, C.J. and Zerwic, J.J. (2014) Fatigue in the Presence of Coronary Heart Disease. Nursing Research, 63, 83-93. http://dx.doi.org/10.1097/NNR.0000000000000019

[7] Ream, E. and Richardson, A. (1996) Fatigue: A Concept Analysis. International Journal of Nursing Studies, 33, 519529. http://dx.doi.org/10.1016/0020-7489(96)00004-1

[8] Brink, E., Karlson, B. and Hallberg, L.R.M. (2002) Health Experiences of First-Time Myocardial Infarction: Factors Influencing Women's and Men's Health-Related Quality of Life after Five Months. Psychology, Health and Medicine, 7, 5-16. http://dx.doi.org/10.1080/13548500120101522

[9] Alsén, P. and Brink, E. (2013) Fatigue after Myocardial Infarction-A Two-Year Follow-Up Study. Journal of Clinical Nursing, 22, 1647-1652. http://dx.doi.org/10.1111/jocn.12114

[10] Alsén, P., Brink, E., Brändström, Y., Karlson, B.W. and Persson, L.-O. (2010) Fatigue after Myocardial Infarction: Relationships with Indices of Emotional Distress, and Sociodemographic and Clinical Variables. International Journal of Nursing Practice, 16, 326-334. http://dx.doi.org/10.1111/j.1440-172X.2010.01848.x

[11] Watanabe, Y. (2008) Preface and Mini-Review: Fatigue Science for Human Health. In: Evengård, B., Natelson, B.H., Jason, L.A. and Kuratsune, H., Eds., Fatigue Science for Human Health, Springer, Tokyo, 5-11.

[12] Maghout-Juratli, S., Janisse, J., Schwartz, K. and Arnetz, B.B. (2010) The Causal Role of Fatigue in the StressPerceived Health Relationship: A MetroNet Study. The Journal of the American Board of Family Medicine, 23, 212219. http://dx.doi.org/10.3122/jabfm.2010.02.090132

[13] Liffman, S., Thorsteinsson, E.B., Brown, R.F. and Hine, D.W. (2012) Mediators and Moderators of Stress-Fatigue and Psychological Distress-Fatigue Relationships in a Non-Clinical Sample. Psychology and Education, 49, 29.

[14] Wikman, A., Messerli-Bürgy, N., Molloy, G.J., Randall, G., Perkins-Porras, L. and Steptoe, A. (2012) Symptom Experience during Acute Coronary Syndrome and the Development of Posttraumatic Stress Symptoms. Journal of Behavioral Medicine, 35, 420-430. http://dx.doi.org/10.1007/s10865-011-9369-X

[15] O’Donnell, S., McKee, G., O’Brien, F., Mooney, M. and Moser, D.K. (2012) Gendered Symptom Presentation in Acute Coronary Syndrome: A Cross Sectional Analysis. International Journal of Nursing Studies, 49, 1325-1332. http://dx.doi.org/10.1016/j.ijnurstu.2012.06.002

[16] Castilla, C. and Vázquez, C. (2011) Stress-Related Symptoms and Positive Emotions after a Myocardial Infarction: A Longitudinal Analysis. European Journal of Psychotraumatology, 2, 8 p. http://dx.doi.org/10.3402/ejpt.v2i0.8082 
[17] Roebuck, A., Furze, G. and Thompson, D.R. (2001) Health-Related Quality of Life after Myocardial Infarction: An Interview Study. Journal of Advanced Nursing, 34, 787-794. http://dx.doi.org/10.1046/j.1365-2648.2001.01809.x

[18] Guler, E., Schmid, J.P., Wiedemar, L., Saner, H., Schnyder, U. and von Känel, R. (2009) Clinical Diagnosis of Posttraumatic Stress Disorder after Myocardial Infarction. Clinical Cardiology, 32, 125-129. http://dx.doi.org/10.1002/clc.20384

[19] Whitehead, D.L., Perkins-Porras, L., Strike, P.C. and Steptoe, A. (2006) Post-Traumatic Stress Disorder in Patients with Cardiac Disease: Predicting Vulnerability from Emotional Responses during Admission for Acute Coronary Syndromes. Heart, 92, 1225-1229. http://dx.doi.org/10.1136/hrt.2005.070946

[20] Sjöström-Strand, A. and Fridlund, B. (2007) Stress in Women's Daily Life before and after a Myocardial Infarction: A Qualitative Analysis. Scandinavian Journal of Caring Sciences, 21, 10-17. http://dx.doi.org/10.1111/j.1471-6712.2007.00433.x

[21] Ginzburg, K., Solomon, Z., Koifman, B., Keren, G., Roth, A., Kriwisky, M., Bleich, A., et al. (2003) Trajectories of Posttraumatic Stress Disorder Following Myocardial Infarction: A Prospective Study. Journal of Clinical Psychiatry, 64, 1217-1223. http://dx.doi.org/10.4088/JCP.v64n1012

[22] Salminen, S., Kouvonen, A., Koskinen, A., Joensuu, M. and Väänänen, A. (2014) Is a Single Item Stress Measure Independently Associated with Subsequent Severe Injury: A Prospective Cohort Study of 16,385 Forest Industry Employees. BMC Public Health, 14, 543. http://dx.doi.org/10.1186/1471-2458-14-543

[23] Lindwall, M., Ljung, T., Hadžibajramović, E. and Jonsdottir, I.H. (2012) Self-Reported Physical Activity and Aerobic Fitness Are Differently Related to Mental Health. Mental Health and Physical Activity, 5, 28-34. http://dx.doi.org/10.1016/j.mhpa.2011.12.003

[24] Jonsdottir, I.H., Rödjer, L., Hadzibajramovic, E., Börjesson, M. and Ahlborg Jr., G. (2010) A Prospective Study of Leisure-Time Physical Activity and Mental Health in Swedish Health Care Workers and Social Insurance Officers. Preventive Medicine, 51, 373-377. http://dx.doi.org/10.1016/j.ypmed.2010.07.019

[25] Elo, A.L., Leppänen, A. and Jahkola, A. (2003) Validity of a Single-Item Measure of Stress Symptoms. Scandinavian Journal of Work, Environment \& Health, 29, 444-451. http://dx.doi.org/10.5271/sjweh.752

[26] Cohen, S., Kamarck, T. and Mermelstein, R. (1983) A Global Measure of Perceived Stress. Journal of Health and Social Behavior, 24, 385-396. http://dx.doi.org/10.2307/2136404

[27] Cohen, S. (1988) Perceived Stress in a Probability Sample of the United States. In: Spacapan, S. and Oskamp, S., Eds., The Social Psychology of Health, The Claremont Symposium on Applied Social Psychology, Sage Publications, Inc., Thousand Oaks, 31-67.

[28] Nordin, M. and Nordin, S. (2013) Psychometric Evaluation and Normative Data of the Swedish Version of the 10-Item Perceived Stress Scale. Scandinavian Journal of Psychology, 54, 502-507. http://dx.doi.org/10.1111/sjop.12071

[29] Smets, E., Garssen, B., Bonke, B. and De Haes, J. (1995) The Multidimensional Fatigue Inventory (MFI) Psychometric Qualities of an Instrument to Assess Fatigue. Journal of Psychosomatic Research, 39, 315-325. http://dx.doi.org/10.1016/0022-3999(94)00125-O

[30] Fredriksson-Larsson, U., Brink, E., Alsen, P., Falk, K. and Lundgren-Nilsson, Å. (2015) Psychometric Analysis of the Multidimensional Fatigue Inventory (MFI-20) in a Sample of Persons Treated for Myocardial Infarction. Journal of Nursing Measurement (in press in Volume 23, Number 1 in April 2015).

[31] Dimsdale, J.E. (2008) Psychological Stress and Cardiovascular Disease. Journal of the American College of Cardiology, 51, 1237-1246. http://dx.doi.org/10.1016/j.jacc.2007.12.024

[32] Steptoe, A., Molloy, G.J., Messerli-Bürgy, N., Wikman, A., Randall, G., Perkins-Porras, L. and Kaski, J.C. (2011) Fear of Dying and Inflammation Following Acute Coronary Syndrome. European Heart Journal, 32, 2405-2411. http://dx.doi.org/10.1093/eurheartj/ehr132

[33] Altman, D.G. (1991) Practical Statistics for Medical Research. Chapman \& Hall, London.

[34] Åkerstedt, T., Axelsson, J., Lekander, M., Orsini, N. and Kecklund, G. (2014) Do Sleep, Stress, and Illness Explain Daily Variations in Fatigue? A Prospective Study. Journal of Psychosomatic Research, 76, 280-285. http://dx.doi.org/10.1016/j.jpsychores.2014.01.005

[35] Hadzibajramovic, E. (2013) Methodological Aspects of the Analysis of Psychosocial Work Environment. Construction of Global Scores and Other Validity Issues Regarding Multi-Item Self-Report Instrument. Licentiate Thesis, Studies from Department of Statistics, Örebro University School of Business, Örebro.

[36] Miaskowski, C., Dodd, M. and Lee, K. (2004) Symptom Clusters: The New Frontier in Symptom Management Research. Journal of the National Cancer Institute Monographs, 2004, 17-21.

http://dx.doi.org/10.1093/jncimonographs/lgh023 